\title{
Multiple Autonomous Discrete Event Controllers for Constellations
}

\author{
Timothy C. Esposito \\ NASA Goddard Space Flight Center, Code 583 \\ Greenbelt. MD 20771 \\ 301-286-8995 \\ timothy.c.csposito@nasa.gov
}

\begin{abstract}
The Muluple Auronomous Discrele Event Controllers for Constellations (MADECC) project is an cffort within the National Aeronautics and Space Administration Goddard Space Fight Centcr's (NASA/GSFC) Information Systems Division to develop autonomous positioning and atritude control for constellation satellites. Il will be accomplished using traditional control theory and advanced coordination algorithms developed by the Johns Hopkins University Applied Physics Luboratory (JHU/APL). This capability will be demonstrated in the discrete event control test-bed located at JYUU/APL.
\end{abstract}

This project will be modeled for the Lconardo' constellation mission but is intended to be adaptable to any constellation mission. To develop a common software archirecture. the controllers will only model very high-level responses. For instance, after determining that a maneuver must be made. the MADECC system will outpul a $\triangle V$ (velocity change) value. Lower level systems must then decide which thrusters to fire and for how long to achieve that $\Delta \mathrm{V}$.
TABLE OF CONTENTS

1. INTRODUCHON: 1

1-1. BACKGROUND .1

1-2. ORGANIZATIONAL ROLES ............................................2

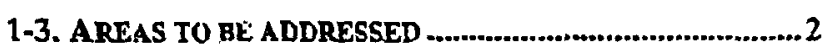

2. MADECC SYSTEMS DEVELORMENT ….............................

2-1. SYSTEM DESCRITTION 3

2.2. RELEASE I - JHU/APL ORGTT DETERMINATION AND PRELIMINARY COORDINATION. 3

2-3. RELEASE II - REORIENTATION ABH-TTY AND ADVANCED MaNFUVER PLANNING. .4

2-4. RELEASE III - INTXLLIGENT COORDDATION AND TASK DISSEMINA TrON.............................................................4

3. TECHNOLOGY DESCRIPTIONS...........................................4

3-1. ORBITAL DYNAMICS .................................................... 4

3-2. CONTROI AND COORDINATION......................................5

4. MADECC FIIGHT POTENTIAL,.....................................5

5. CURRENT PROGRESS..............................................................6

\section{INTRODUCTION}

\section{1-1. BACKGROUNn}

Salellite orbit and attitude derermination lasks have traditionally been performed on the ground. Commands were then uplinked to the satellite to relay the pre-computed information. Historically, the lack of tlight CPU processing power and the complexity of required algorithms have precluded the application of anboard orbit and attitude detcrmination.

However, ground-based orbit and attitude functions often result in large manpower expenditures, and rely on limited communication. When multiple satellites are introduced to the system, these problems are magnified. Moving the orbit and attitude dctermination tasks onboard the spacccraft

${ }^{1}$ Governmeal work not prolected by U.S. 
would be a huge benefit, especially if the satellites could coordinate among themselves. As flight CPUs and software algorithms improve, fully automated constellation management will become possible. The MADECC project is a demonstration showing the feasibility of moving such tasks onboard the spacecraft, and automating the necessary coordination and control within the constellation structure.

When orbit and attitude determination are moved to the spacecraft and become automated, other benefits emerge. For instance, a constellation may have the ability to recognize an unexpected, scientific target of opportunity. Instead of waiting for the satellites to be in view of a ground station or the space network for commanding, the constellation can reorient itself automatically to ensure exploitation of that opportunity.

This ability to locate scientific targets of opportunity will also enable deep space constellation missions. Data rates for deep space satellites can be on the order of bits per second, where commanding can be delayed and difficult. If an unexpected event has occurred in deep space, personnel on the ground are not notified quickly, the opportunity to start collecting information on that event will be missed. In addition, the reduced communication needs allow the bandwidth back to earth to be reserved for science data only, thus maximizing science benefits.

The Leonardo mission will take measurements of solar radiation that is reflected off the Earth's surface from different angles simultaneously. Due to varying contours and surfaces of the Earth, its magnetosphere is not uniform. Scientists do not fully understand yet how solar radiation is reflected and how it affects the Earth's magnetosphere. Solar radiation reflection back into space can vary drastically at different locations on the earth, especially over deserts, oceans, mountains, volcanoes, and even large fires. Prior missions have taken many single point measurements, but this type of measurement limits our ability to fully understand the nature of the solar radiation reflectivity in three dimensions. To understand these properties, measurements must be taken from multiple angles. Six satellites will fly in the Leonardo constellation to accomplish this task ${ }^{2}$. Maximum efficiency orbits, shown in Figures 1-1 and 1-2, have been designed by GSFC's Flight Dynamics Analysis Branch (FDAB). This scenario will be used as the initial MADECC development and test scenarios.

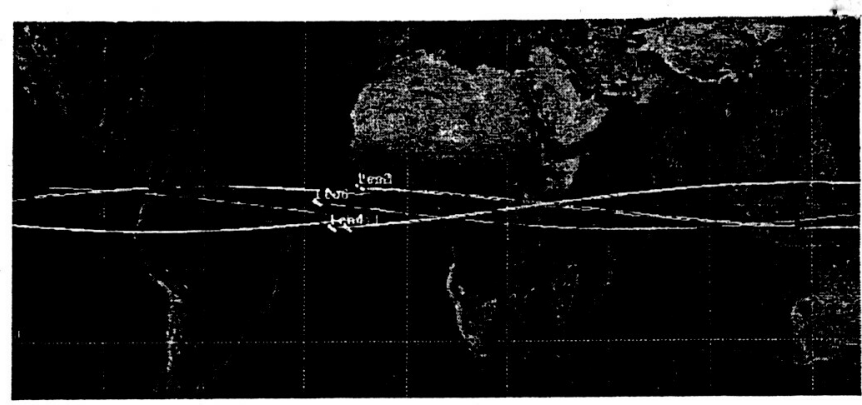

Figure 1-1 - Ground Track for Leonardo Constellation

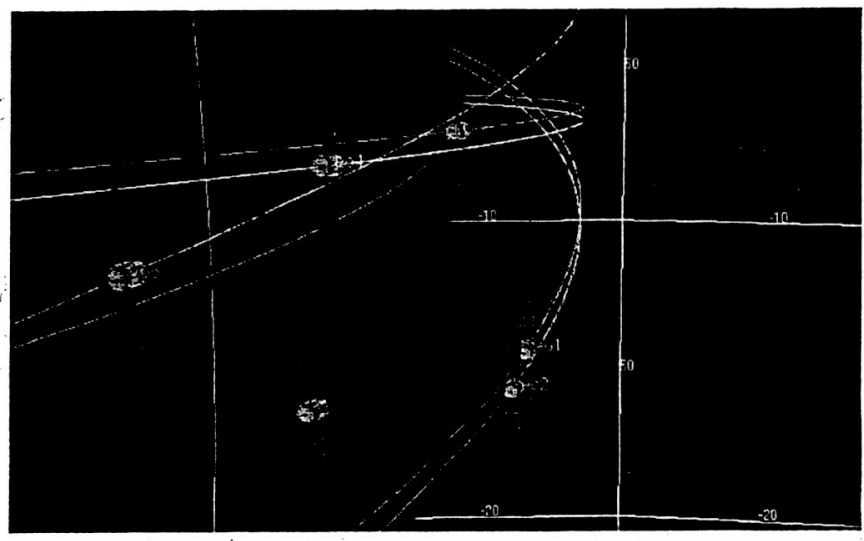

Figure 1-2 - 3D View of Leonardo Constellation Arrangement

\section{1-2. ORganizational Roles}

The NASA/GSFC Mission Applications Branch will develop the orbital dynamics portion of the system, implement the control and coordination algorithms, and construct the overall MADECC software systems. JHU/APL will provide the control and coordination algorithms, and also houses the test-bed on which the MADECC system will be demonstrated. The NASA/GSFC FDAB will provide analysis and navigation and control expertise. Additionally, there are plans to integrate the controllers with the GSFC Formation Flying Test-bed.

\section{1-3. AREAS TO BE ADDRESSED}

Several key areas require attention when designing onboard discrete event controllers for multiple satellites. Those areas include:

- Orbit determination

- Attitude determination

- Maneuver planning

- Cross-link communication

- Satellite coordination

The orbit determination problem is one of the key issues that must be addressed. Simply stated, the satellite cannot make decisions of what to do if it does not know its position 
or velocity. The Global Positioning System (GPS) Enhanced Orbit Navigation System (GEONS) software will initially provide the orbit determination for the MADECC system. GEONS provides a highly accurate and autonomous orbit determination solution for low-Earth and high-Earth orbiting satellites, using measurements derived from GPS, GPS Wide Area Augmentation System (WAAS), inter-satellite, ground station to satellite, and Tracking Data Relay Satellite System (TDRSS), forward-link signals, and Sun and Earth horizon sensors.

Traditionally, attitude systems require significant customization for every mission. At least in the near future, this will continue to be the case. There may even need to be significant customization for each satellite attitude system in the constellation, depending on mission goals and spacecraft designs. The Leonardo mission will be a relatively simple attitude problem to solve since every satellite will focus on the same point when collecting data, allowing each satellite to use the same logic.

The maneuver planning function will require inputs from the orbit and attitude determination functions to determine an efficient plan for relocating orbit and/or changing orientation.

The cross-link communication area has been extensively addressed by JHU/APL. They have developed their own cross-link transceiver, which is already integrated into their test-bed.

Additionally, they have researched many coordination schemes for communication among the satellites ${ }^{1}$. Potential candidates include differing levels of a master/slave relationship, or having all satellites maintaining equal knowledge of every other satellite. Each scenario has its advantages and disadvantages. For this project, the most appropriate coordination scheme for the Leonardo mission will be identified and implemented.

\section{MADECC SYSTEMS DEVELOPMENT}

\section{2-1. SYSTEM DESCRIPTION}

The MADECC system consists of several major software modules, which correspond to the system functionality. Figure 2-1 shows the interfaces between these functions. The MADECC modules are shown as light gray ovals, and the system manager is shown in the center as a light gray rectangle with shadowing. External interfaces are displayed as light green diamonds.

All modules will be coded as plug-and-play components, as missions other then Leonardo may require the inclusion of alternate modules. For instance, a deep-space mission would require a different orbit determination module, since GEONS only currently provides an orbit solution for an Earth orbiting satellite.
It is very important to note that the MADECC system will take advantage of as many existing developed technologies as possible. Since the system handles orbit determination, attitude control, maneuver planning, coordination, crosslinking, and telemetry handling capabilities, the development of all functionality from scratch would require significant time and manpower. By taking advantage of existing work such as the coordination algorithms developed by JHU/APL, FDAB's GEONS, and AutoCon ${ }^{\mathrm{TM}}$ F developed by NASA/GSFC and ai solutions, Inc., MADECC plans to integrate these systems to accomplish tasks of greater complexity and larger scope.

The major functionalities of the MADECC system will be developed in three major releases, each with a specified goal and a definitive deliverable product.

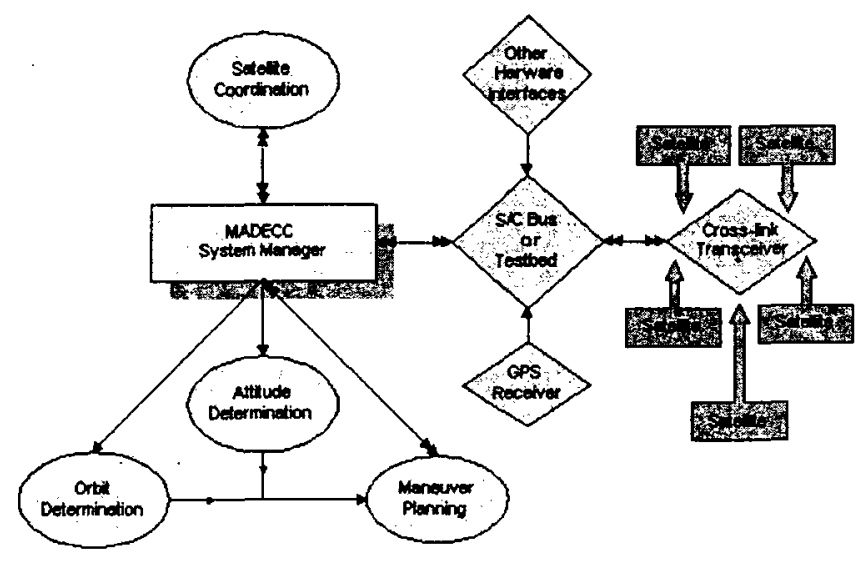

Figure 2-1 - MADECC System Configuration

\section{2-2. RELEASE I - JHU/APL ORBIT DETERMINATION AND PRELIMINARY COORDINATION}

The goal of release I is to demonstrate the ability to maintain six individual orbits of the Leonardo constellation and have each of the satellites notify the others of its status via the satellite coordination module. This will result in all satellites having full awareness of total constellation health. A protocol for transmitting data to others in certain precedence order will be developed. This protocol will need to address issues that focus on the minimum amount of required data transmissions between satellites. Each data communication must leverage off previous communications to ensure that bandwidth and power are not wasted. The deliverable product from this release will demonstrate preliminary coordination abilities. Since the JHU/APL testbed is already capable of determining an orbit position and velocity and transmitting truth-values, those values will be obtained directly from the test-bed in release I.

The following functions will be developed for release I:

- MADECC System Manager (partial)

- Maneuver Planning (partial)

- Satellite Coordination (partial) 
Completion of the release I product is planned for January 2003.

\section{2-3. RELEASE II - REORIENTATION ABILITY AND Advanced Maneuver PlanNing}

Release II will fine-tune the release I modules and add the following new capabilities:

- MADECC System Manager (partial)

- Orbit Determination (GEONS)

- Attitude Determination

- Maneuver Planning (full)

The maneuver planning module will need extensive advancements. It will need to accept inputs from both the orbit determination and attitude determination modules, and develop an intelligent plan to satisfy requirements that sources be as efficient as possible. In this release, there are plans to integrate the GEONS software for an alternate method of orbit determination. This will allow simpler integration with other test-beds that lack existing orbit determination capabilities.

The major goal of release II is to demonstrate the ability to control attitude and to plan maneuvers. The release II product is scheduled for completion in September 2003.

\section{2-4. RELEASE III - INTELLIGENT COORDINATION AND TASK DISSEMINATION}

The release III goal is to develop true intelligence of satellite coordination into the discrete event control system. The following functionality will be added:

- MADECC System Manager (full)

- Satellite Coordination (full)

In particular, the satellite coordination module will need significant enhancements. The objective is to have each node in the constellation understand its capabilities. A mission can then define its goals, and the satellites will coordinate among themselves to disseminate the identified tasks to those capable of completing them. Following this scheme provides essential flexibility. As a node in the constellation degrades, its capabilities may also decrease. For instance, a sensor may become dirty and no longer usable, requiring the task to collect that type of sensor data to be assigned to another node, therefore maintaining the mission goals.

A demonstration of these capabilities is planned for September 2004.

\section{TECHNOLOGY DESCRIPTIONS}

\section{3-1. ORBital DYNamics}

The logic of calculating the spacecraft equations of motion and determining current and predicted positions and attitude is a separate function from that of the system coordination. The system that holds the coordination intelligence is configured to understand specific constraints. The maneuver planning module will rely on the AutoCon ${ }^{\mathrm{TM}}-\mathrm{F}$ package to execute the GSFC algorithm or the JPL algorithm. It was developed for the EO-1 mission to autonomously maintain a formation-flying pattern with Landsat-7. AutoCon ${ }^{\mathrm{TM}}-\mathrm{F}$ is capable of accepting the current and desired positions as inputs, and providing a propagated state without a maneuver and a maneuver plan to reach the desired position. Based on the constraints set in the control scenario, the goal is to have the control scenario accept the propagated state without a maneuver. If that does not meet the constraints within the control set, the $\Delta V$ values retrieved from AutoCon ${ }^{\mathrm{TM}}-\mathrm{F}$ will become an input to the satellite coordination module, which will then coordinate the maneuvers between the satellites.

The first Leonardo autonomous control scenarios will focus on a very simple case. To keep the scenarios as simple as possible, only two types of maneuvers will be allowed: a Hohmann Transfer, or orbit transfer, and a plane change. It is well known that a direct plane change is not the most efficient maneuver when done alone, but it will provide for a very simple demonstration case of control and coordination among satellites in a constellation.

The Hohmann Transfer is the most efficient orbit transfer method when no other maneuvers are needed. Future releases of MADECC will contain logic for combining various maneuver methods. As shown in the equations below, it is a straightforward method to follow for calculating the $\Delta \mathrm{V}$ values needed to transfer to another orbit. $^{5}$

$$
\begin{gathered}
\Delta V_{L}=\sqrt{\mu} \cdot\left[\left(\frac{2}{a_{L}}-\frac{1}{a_{T}}\right)^{1 / 2}-\left(\frac{1}{a_{L}}\right)^{1 / 2}\right] \\
\Delta V_{H}=\sqrt{\mu} \cdot\left[\left(\frac{1}{a_{H}}\right)^{1 / 2}-\left(\frac{2}{a_{H}}-\frac{1}{a_{T}}\right)^{1 / 2}\right] \\
T=\frac{1}{2} \cdot P_{T}=\pi \cdot a_{T}^{3 / 2} \cdot \mu^{-1 / 2}
\end{gathered}
$$

where ...

$$
\begin{gathered}
a_{T}=\frac{1}{2} \cdot\left(a_{L}+a_{H}\right) \\
a \equiv \text { semi-major axis } \\
\Delta V \equiv \text { change in velocity } \\
\mu \equiv \text { gravitational constant }
\end{gathered}
$$




\section{$T \equiv$ transfer time \\ $P \equiv$ orbit period \\ $L$ subscript refers to lower circular orbit. \\ $H$ subscript refers to higher circular orbit. \\ $T$ subscript refers to Hohmann transfer orbit.}

As shown, it is a straightforward solution to plan an orbit transfer with the above equations. The process is composed of having two impulsive burns. The first burn is used to go into an elliptical transfer orbit, with the satellite staying at that transfer orbit for a length of time $T$. At the end of that time, another burn will occur to direct the satellite in the right path of the final orbit.

The case of planning an orbit change is also quite simple, comprising a simple vector addition of the initial and final states. $^{5}$

$$
\Delta V \angle \theta=\left(V_{i}^{2}+V_{f}^{2}-2 \cdot V_{i} \cdot V_{f} \cdot \cos \theta\right)^{Y / 2}
$$

where ...

$$
\begin{gathered}
V_{i} \equiv \text { initial velocity } \\
V_{f} \equiv \text { final velocity } \\
\theta \equiv \text { change of angle }
\end{gathered}
$$

Once again, in ordinary scenarios, more logic would take place to plan for an efficient burn. In the future, many more solutions will be evaluated for repositioning, such as combined maneuvers, 3-burn transfers, and aero-assists trajectories. Plane changes are $\Delta V$ expensive maneuvers in nature, especially at lower altitudes where initial velocities are higher. Logic is used in the early stages to keep the coordination schemes simple at first, since they can quickly become complex and too burdensome for early demonstrations.

\section{3-2. CONTROL ANd COORDination}

The key to autonomous control of the entire constellation is the ability to coordinate satellite actions amongst themselves. Each individual satellite must not only be knowledgeable about itself, but also knowledgeable about the other nodes in the constellation, and must understand where it fits into the overall mission goals. This is both a logically and computationally difficult task that cannot be addressed by traditional control theory. For a single-node system acting by itself, the properties ordinarily examined are response time, stability and robustness. For multiplenode system, the required set of parameters to monitor expands to include dynamic system behavior adaptation, coordination of autonomous system assets, goal planning and refinement, fault resolution, and learning. ${ }^{3}$ The expanded requirement demands more intelligent control techniques, such as neural networks, fuzzy logic, discrete event systems, and expert systems. ${ }^{4}$

The MADECC system will take advantage of discrete-event systems and model-based reasoning to effectively control the formations and accomplish the mission goals. Discrete- event systems are composed of a discrete state space that contains every system configuration and state transition structure that defines how the system's state-space evolves with time. ${ }^{4}$ The discrete event system will be represented with finite state automata that can be defined with a formal language construct. Using finite state automata is a way of deciding whether a defined state is acceptable or not through the use of pattern recognitions with a finite set of states. With the finite state automata outputs, the system will be aware of its current situation. That knowledge can then be fed into a model-based reasoning system to make further decisions of what must occur next. Massachusetts Institute of Technology, working jointly with JHU/APL, developed the model-based reasoning system to be used in the MADECC system.

The satellite coordination module will gather information from the cross-link transceivers while communicating with the other satellites. It then uses that information to decide whether an action must be taken to accomplish the mission goals. The high-level decisions, such as "All satellites need to be $1000 \mathrm{~km}$ apart from one another", will come from outputs of the model-based reasoning system based on rules that the MADECC system manager define. If the satellite coordination module concludes that an action must be taken, then the satellites begin the process of communication and negotiation.

To reposition the satellites, one or more maneuvers may be needed. As discussed previously, these details of required maneuvers will come from the maneuver planning module. One satellite will be responsible for making the high-level decisions, and it will be up to each individual satellite to calculate the details of its own maneuver plan. Given current flight processors, it would also be impossible for a single satellite to plan maneuvers for all satellites in the constellation. Once a total plan of action has been established, the satellite coordination module will pass a maneuver token to allow an individual satellite to perform its maneuver. Once completed, it will be responsible for contacting the other nodes to report on its status, providing the most efficient use of constellation resources.

In future scenarios, it will be necessary for multiple spacecraft to perform maneuvers simultaneously. The same concepts previously described can be adapted to this situation as well. Multiple maneuver tokens may be passed around, and it will be the responsibility of the master spacecraft to make sure the entire constellation is aware of the constellation status.

\section{MADECC Flight Potential}

The MADECC system is a prototype system to demonstrate a new technology, but the inclusion of existing flight-ready components will lay the groundwork for possible evolution to flight embedded software. The JHU/APL cross-link transceiver already has the ability to provide a smoothed orbit determination solution with software embedded in the 
transceiver itself. AutoCon ${ }^{\mathrm{TM}}-\mathrm{F}$ is currently being flown on EO-1, and GEONS is flight-tested code as well. GEONS will provide an alternate method of obtaining an orbit determination solution. The selection of ANSI C and C++ as implementation languages will aid in the development of a system that is operating system independent.

Additionally, the main components of the MADECC system have been designed as separate software modules, allowing components to be easily replaced.

\section{CURrent Progress}

This project is currently in its development phase. The MADECC system is now capable of processing position, velocity, acceleration, quaternion, and orbital events data, displaying that data in a separate interface application, displaying spacecraft ground tracks in Satellite Tool $\mathrm{Kit}^{\mathrm{TM}}$ (STK), and transmitting information among satellites through the cross-link transceiver. The coordination and control scenarios are currently being devised and implemented. Once that step is complete, responses to achieve desired behaviors and performance will be analyzed. To analyze responses, the following items will be evaluated:

- Deviations from predicted orbits

- Time to communicate planned maneuvers among spacecraft

- Time to return to predicted or planned orbits

- Settling times

- Damping ratios

- Time available for science

- Overall formation behavior

To analyze performance, the following items will be examined:

- Time to communicate data through cross-links

- $\mathrm{CPU}$ and memory measurements for coordination and planning tasks

- Time to complete planned maneuvers

- Fuel efficiency of coordinated maneuvers

After response and performance analysis is complete for one scenario, an optimization activity will occur.

Autonomous positioning control, attitude control, and coordination will be demonstrated and shown to be possible once the analysis described above is taken. The MADECC project will hopefully prove to be a stepping-stone to autonomous control and coordination of multiple spacecraft missions.

\section{REFERENCES}

[1] Angelopoulos, V., and P. V. Panetta, eds. Science Closure and Enabling Technologies for Constellation Class Missions. University of California, Berkeley and NASA, Goddard Space Flight Center, p. 29, 1998.

[2] Bristow, John, Carol Raymond, and Mark Schoeberl. Intelligent Distributed Spacecraft Infrastructure. Earth Science Enterprise Technology Planning Workshop, p. 7, 2001.

[3] P. A. Stadter. Discrete event command and control for formation flying of distributed small spacecraft systems. 13th Annual AIAA/USU Conf. on Small Satellites, SSC99-VI-4, 23-26 August, Logan, UT, 1999.

[4] P. A. Stadter, G. R. Barrett, D. P. Watson, T. C. Esposito, and J. O. Bristow. Autonomous command and control for distributed spacecraft systems. AIAA Nanotech 2002 Proceedings, Houston, TX 9-12 September 2002.

[5] Wertz, James R. Mission Geometry; Orbit and Constellation Design and Management. Microcosm Press, El Segundo, CA and Kluwer Academic Publishers, Dordrecht, Netherlands, 2001.

Timothy C. Esposito is a computer engineer working in the Mission Applications Branch (Code 583) at NASA Goddard Space Flight Center. He received his degree in aerospace engineering at North Carolina State University. At NASA, he has helped to develop

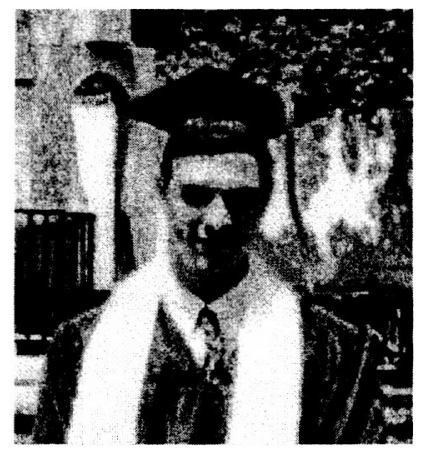
and maintain the flight dynamics software systems for the EOS Terra, EOS Aqua, EO-1, and SMEX missions. His focus has been on developing autonomous software in the flight dynamics arena. 